\title{
Redefining Requirements of Ancillary Services for Technology Agnostic Sources
}

\author{
D. E. M. Bondy, O. Gehrke, \\ K. Heussen, H. W. Bindner \\ Technical University of Denmark \\ \{bondy,kh,olge,hwbi\}@elektro.dtu.dk
}

\author{
E. C. Kara, D. P. Chassin, S. Kiliccote \\ SLAC National Accelerator Lab \\ \{ekara,dchassin,silak\}@slac.stanford.edu
}

\begin{abstract}
New sources for ancillary services are needed, yet the requirements for service provision in most countries are explicitly formulated for traditional generators. This leads to waste of the potential for new technologies to deliver ancillary services. In order to harness this potential, we propose to parameterize the requirements of ancillary services so that reserves can be built by combining the advantageous properties of different technologies. The proposal is exemplified through a laboratory test where it shown that the system needs can be covered through cheaper and smaller reserves.
\end{abstract}

\section{Introduction}

Ancillary services (AS) are essential for the reliable operation of power systems. In some countries, the high penetration of renewable energy sources is leading to the decommissioning of traditional generators, thus reducing the available resources for AS provision [1]. With the increase in adoption of distributed energy resources (DERs) and controllable smart loads, as well as the emergence of schemes for utilizing consumption flexibility, such as demand response (DR), new sources for AS from the demand-side are potentially available.

Demand-side resources (DSRs) possess qualities that in many cases match the performance needs of the system better than traditional generators [2], yet the requirements for AS in many countries are defined on the implicit assumption that only generators provide ancillary services. Therefore, these requirements can be a barrier for the participation of DSRs in the AS markets [3]. There are both economic and technical benefits in exploiting the qualities of flexible demand.

This work parametrizes the requirements for resources providing AS such that the reserve can be sized optimally to the overall system requirements. The overall system requirements are also redefined in terms of a desired shape instead of a capacity. The new parametrized requirements are focused on service performance and are source/technology independent. By changing the AS requirements to focus on performance rather than unit capabilities and utilizing new technologies as AS sources, system operators may increase sys-

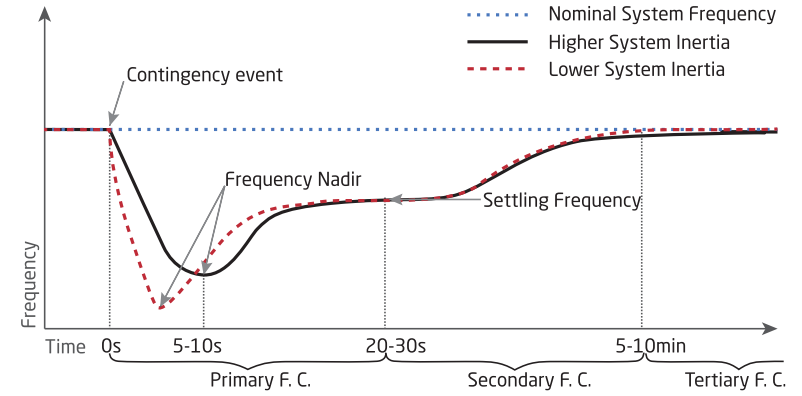

Figure 1: System frequency following a generation contingency event.

tem reliability [4], reduce the size and cost of the reserve, and increase participation in the AS markets.

The rest of the paper is organized as follows: Section 2 reviews the current state of power system operation, ancillary service definitions and requirements; Section 3 presents the problems and opportunities we address. Section 4 formulates new AS requirements and an exemplary AS market clearing setup; Section 5 presents a case study of the impact of the new requirements, and section 6 presents conclusions and future research paths.

\section{Ancillary Services from Demand Re- sponse}

In electric power systems, supply and demand must be kept balanced at all times while respecting network limitations and operational constraints. For unanticipated imbalances, system operators procure specific Ancillary Services as operating reserves to respond when these imbalances manifest. A common metric used to indicate imbalance in a power system is the system frequency.

Figure 1 illustrates an imbalance, possibly the result of a contingency event like a generator unexpectedly going off-line, and the resulting frequency time series as the system responds with its ancillary services.

\subsection{Power system operation and ancillary ser- vices}

In Figure 1, a contingency event occurs which causes the system frequency to fall. Operating reserves begin 


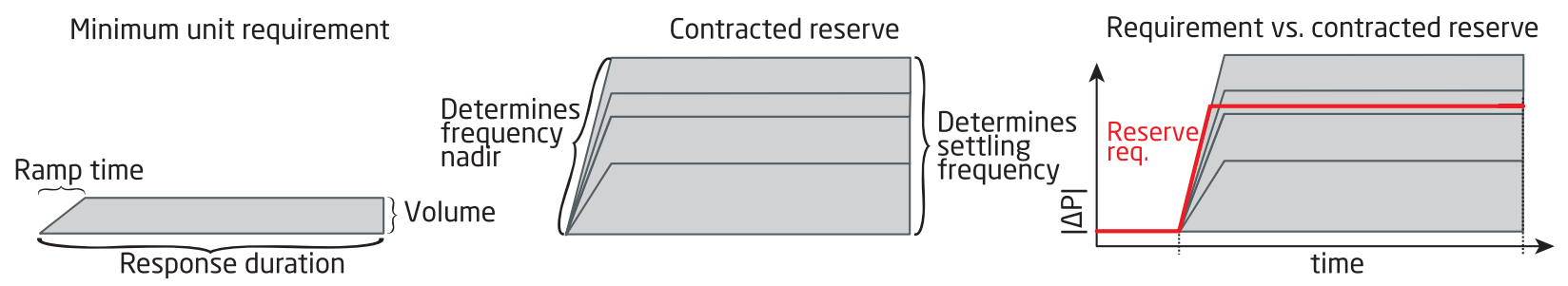

Figure 2: Reserves have traditionally been sized using offline studies of frequency response (both nadir and settling frequency) to $N-1$ contingencies.

to respond to arrest the frequency excursion and then return system frequency to its nominal value. While the structure of ancillary services differs between systems, generally there are the following three categories of response, matching the AS framework presented in [5].

Primary reserves, also called frequency droop control, and in ENTSO-E terms 'Frequency Containment Reserves' [6], are the first and fastest with a response time in seconds. Primary reserves are resources controlled locally, responding directly to local measurements of the system frequency. They arrest the falling frequency and begin to restore it towards a settling frequency corresponding to the physical imbalance.

Secondary reserves, also called 'regulation' or 'area control' (in ENTSO-E terms 'Frequency Restoration Reserves') operate in the seconds to minutes time frame. These reserves are activated via a communication based (centralized) control scheme to release active primary reserves and to return the frequency to its nominal value, respectively re-balance the .

Finally, tertiary reserves are slower, often manually activated resources, also called 'load following' in US; in ENTSO-E terms 'Replacement Reserves'. These reserves are employed to return the system back to a stable state and restore the fast acting reserves while converging back to the nominal system frequency.

Secondary reserves, and in some places also primary reserves, are procured through market mechanisms on a daily basis. The quantity of reserves that a system operator will procure is based on a pre-defined reserve requirement that should provide adequate response capability for any possible single contingency event in a system $[7,8]$. The work presented here explores an alternate reserve requirement definition based on the shape of system response needs and presents a procurement mechanism to limit the over-provision of reserves.

\subsection{Service requirements and limitations}

Until now, system operators have been able to arrest frequency excursions fast enough because of the inherent system inertia. But as the inertia decreases, system op- erators require faster response times from primary frequency control (as illustrated in Figure 1).

The system operator must have sufficient primary reserves to arrest the frequency as fast as possible, before the system enters a state where a blackout is inevitable. A metric for the sufficiency of procured reserves is the frequency nadir [9], illustrated in Figure 1, and it is desirable to keep it as close to the settling frequency as possible. Similarly, the system operator ensures that the secondary reserves act as fast as possible to relieve the primary reserves and also bring the frequency from the settling frequency back to the nominal frequency.

Because AS are essential for the secure operation of the system, the system operators also have requirements and restrictions on the units providing AS. A super-set of requirements across different systems has been identified in [5]. Most service requirements are oriented towards the least common denominator of service providers, e.g. a unit providing primary frequency control in Denmark should provide half of the service within 15 seconds, full response within 30 seconds, and sustain it for $15 \mathrm{~min}$ utes [10]. A system operator acquires a reserve based upon the assumption that all units have the same minimum characteristics. We illustrate this in Figure 2, where the left most block is a geometrical representation of the response characteristics (ramp rate, duration of response and volume) of, e.g., a single synchronous generator. The contracted reserve is a stacking of the assumed responses of the units, which must satisfy the reserve requirement. Traditionally, this dimensioning has been done based upon a desired settling frequency, frequency nadir and the $n-l$ criteria. Figure 2 shows that the reserve must be over-dimensioned (in terms of volume) if the stacked response is to fulfill the ramping requirements that ensure an acceptable frequency nadir.

In short, the historical definition for service requirements has an implicit bias for traditional resources. Alternative technologies are restricted in their contribution to AS provision and their favorable properties are not utilized or undervalued. 


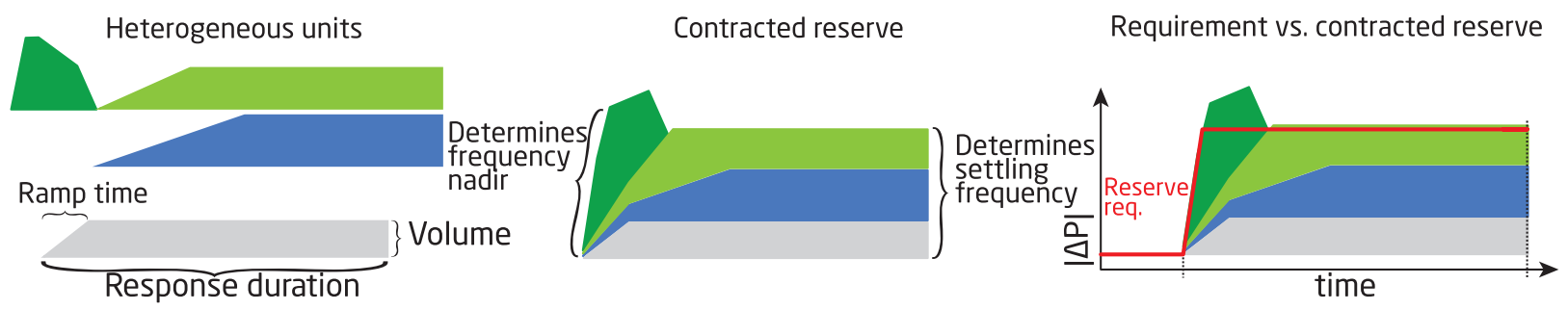

Figure 3: System operators can secure reserves by procuring them based upon mixed resources capabilities.

\section{Problem Statement}

Although DSRs provide additional freedom to help shape response compared to traditional AS providers, the existing AS market rules and requirements are a strong barrier to DSR market participation. A recent study identifies such barriers in the US [3]. The rules and requirements that limit resource participation in different markets are not consistent among different RTOs and ISOs; however, the authors identify three major groups of these rules: rules on the size of the resource, rules on the measurement and telemetry of the resource, and rules on market bidding time. Out of six different ISOs and RTOs in the US, only one allows load aggregation to provide regulation services, and only two allow aggregation participation as a spinning reserve provider. Furthermore, only two ISOs and RTOs allow aggregate telemetry. Providing telemetry at an individual resource level increases the overall cost of metering, making it challenging for DSRs to provide cost-competitive AS.

Similarly, [11] shows the current status of the regulatory condition for DR in Europe. It states that, although great advances have been made towards opening the markets to DR, there are still many regulatory barriers. The report classifies these barriers in four groups: 1) demand response access to market, 2) service provider access to market, 3) product requirements, and 4) measurement and verification, payments and penalties.

The ideal resource to satisfy the system need is one which (1) is always available, and (2) always capability exceeds the ramp rate, power capacity and energy loss of all the resources for which it must compensate. In principle, load is an ideal resource since (1) the load is always present, and (2) it is always greater than or equal to the supply that might be lost.

In [12] it is shown that if primary frequency response is provided by demand response (with a very fast response), the frequency nadir occurs at higher frequencies. In [13], the authors argue that the value of regulation resources can be defined based upon the ramp capabilities of the service providing units. Following the conclusions presented in $[12,13]$, an optimal utilization of demand-side resource technologies for AS delivery could lead to arresting frequency excursions at higher frequency nadir, thus lessening the required amount of supply-side reserves, and thus overall a lower system operation cost.

From an economics point of view, two methods can be utilized to include and incentivize the participation of technologies that in some respects are closer to the ideal than those defined by the current service and market requirements: product differentiation and product restructuring.

Some transmission system operators, like PJM, have implemented the FERC pay-for-performance requirement by differentiating their regulation AS into two products, RegA and RegD [14], splitting their regulation market into a slow service product and a fast service product.

This work explores the alternative: restructuring the market so that all technologies can participate in the same market, and the system operator can optimize the use of the resources based upon their capabilities. This entails reformulating the performance requirements, and removing the requirements that implicitly assume that the services are provided by traditional generators, thus making the requirements technology-agnostic. This would lead to a reserve procurement as shown in Figure 3 .

\section{Restructuring the Ancillary Service Re- quirements}

A stated previously, our objective in this paper is to discuss and formulate requirements for $\mathrm{AS}$ in a number of relevant features, and to provide a market clearing mechanism that selects a portfolio of resources limiting overprovision of reserves. In order to do this, we propose that the market mechanism operates in a resource-agnostic and performance-oriented way. By doing so, we propose a strategy in which (i) we remove the barriers preventing unconventional resources from participating in AS markets due to the static nature of AS market definitions and requirements, and (ii) we provide a fair and performancebased market clearing structure in which the unused po- 
tential of DSRs (and other technologies) is utilized.

\subsection{Overall approach}

The proposed restructuring assumes that system operators acquire AS reserves through a market, and that potential AS providers bid their reserve capacity in that market. The restructuring is based on the following four key concepts (which are further developed in this section):

- The formulation of an ideal ancillary service reserve that the system operator desires for the system will depend strongly on system needs, e.g. a reserve with very fast response in case of low system inertia, and will be submitted as a tender offer to the market.

- The parameterization of the AS bids, where the parameters reflect the service providers' ability to perform as an ideal reserve, removes the minimumrequirements-barriers on new technologies, thus enabling any potential useful unit to participate in the AS provision, which facilitates market liquidity and reduces overall costs of acquiring and calling on needed resources.

- Clearing all units under a generalized single clearing-price auction provides incentives to bid actual marginal cost. In this auction, the capability value of each service provider, their availability and their historical performance is taken into account.

- Performance-based remuneration creates an incentive to provide better AS resources and enables transparent performance-based clearing of the market.

Based on an assessment of the complete decision process, we merge the four key concepts outlined before into a novel approach to AS valuation that accounts both for resource performance and the actual spectrum of system needs. The holistic assessment includes:

- Planning: Parameterization of resource performance and specification of tender conditions based on the assessment of system needs;

- Scheduling: Quantification of AS tender volume, AS bid submission, and market clearing;

- Operation: Monitoring the performances of reserve dispatch/activation based on the traditional definitions related to frequency excursions; and

- Settlement: Verification of service delivery and remuneration.

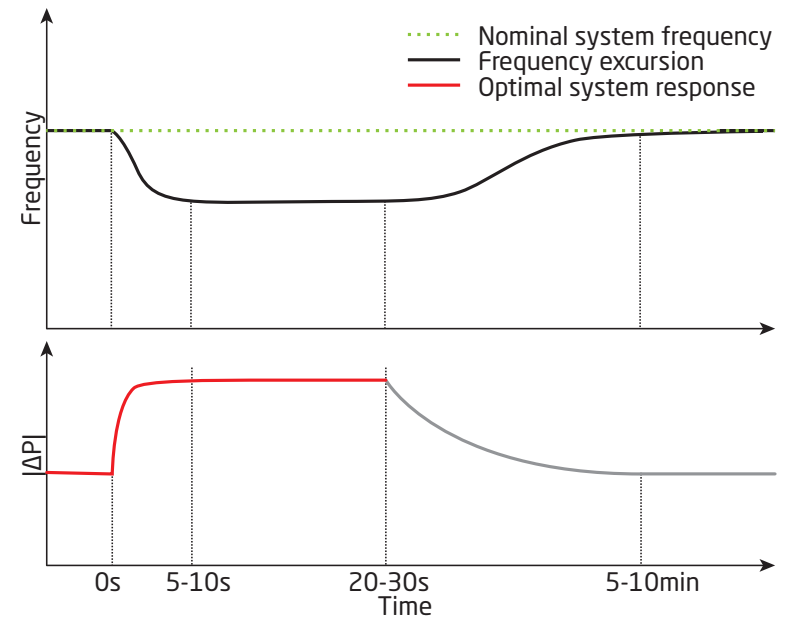

Figure 4: The ideal ramp response is determined mainly by system inertia and sustained until secondary frequency control is activated.

Our proposal focuses on a new parametrization of services (Sec. 4.3), which affects in particular market clearing (Sec. 4.4) and remuneration (Sec. 4.6).

\subsection{Ideal service tender}

In existing AS, there is an implicit assumption that ideal unit response corresponds to a scalar fraction of the required system response (see Sec. 2.2). Therefore, a fast response of individual units is required. In the presence of a diverse resource portfolio, a better system response can be achieved, e.g. by combination of a fast duration-limited and slower unlimited response time resources. The fast responding units become part of an overall cheaper mixed portfolio, which delivers a better system response.

For example, a system operator could determine that the ideal system response to a frequency excursion is the one that has a resulting frequency nadir at the settling frequency (thus minimizing the risk of tripping the underfrequency relays). Based upon the inertia of the system, the system operator determines the volume $\left(V_{t o t}\right)$ needed as well as the response characteristics needed to achieve this, see Figure 4.

\subsection{Parametrization of service performance}

Today, AS requirements are not differentiated according to the capabilities of the unit providing the service. Therefore, service definitions are designed to accommodate the least capable unit in the portfolio. As a consequence, more capable units are not being fully utilized, leading to excess contracting of service providers. 
This sub-optimal allocation of resources could be addressed by introducing a performance dependent definition of AS, i.e., a service definition that allows compliance to be measured on a linear rather than a binary scale: In addition to compliance and noncompliance, different levels of partial compliance are possible. In this context, services will be defined such that the best possible performance of the most capable unit corresponds to full compliance.

One of the challenges with such an approach is to achieve a useful definition of partial compliance. Depending on the complexity of the service, many parameters of DR resources may have to be included in a performance comparison to determine their relative value. For example, resources with identical response magnitudes, ramp rates and duration may represent a significantly different value to the buyer of a cyclic service if one resource requires a longer recovery time between cycles than the other. A performance model is therefore needed to provide a mapping between the multidimensional parameter space of a DR resource and the degree to which it fully satisfies the needs of the TSO, expressed on a linear scale.

We introduce the following definition of a capability value:

$$
\kappa_{i}=g(\mathbf{x}) \quad \in[0,1]
$$

where $g$ is a functional mapping the parameter space $\mathrm{x}$ to a scalar value according to resource utility. This mapping function is highly specific to a particular service and must therefore be developed with care by the service requester; e.g., a TSO, in order to avoid units with certain capabilities gaining too much market power.

The functional $g$ is communicated to the resources as part of the service definition included in a tender. The scalar value $\kappa$ for a particular resource can then be calculated by its operator prior to bidding.

\subsection{Market mechanism}

The market mechanism progresses as follows: the TSO identifies a tender request $T_{\text {tot }}=f(\mathbf{x})$, where $f$ maps the parameter space $\mathbf{x}$ to the desires reserve requirements, and the mapping $g$ of the individual bid to $\kappa_{i}$ is announced for market participants. The tender request can be mapped to a piecewise linear response function $V_{T S O}$ that is depicted by the red line in Figure 3.

The TSO transforms the market participants' bids into a similar piecewise linear response function:

$$
V_{i}(t)=h(\mathbf{x}),
$$

where $h$ is a function that maps the parameter space to the piecewise linear response. The stacking of all $V_{i}(t)$ must fulfill the reserve requirement $V_{T S O}$ :

$$
\sum_{i \in \Omega^{a c c}} V_{i} \geq V_{T S O}
$$

where $\Omega^{a c c}$ is the pool of accepted bids.

In order to leverage the proposed AS restructuring, the market clearing mechanism needs to be adapted. One option is to take the capability value of the service providers, $\kappa_{i}$, a historical service performance index, $\gamma_{i}$, and a unit reliability index, $\epsilon_{i}$, into account. There are many different ways of formulating such a market clearing mechanism. We present an example of a market that utilizes the service parameterization to form an ideal service response at the end of this section.

\subsection{Reserve activation and operation}

The specification of tender and bid parameterization needs to be aligned with the activation mechanism applied during real-time operation of the resource dispatch and activation. For example, fast activation upon detection of frequency excursion is important for primary frequency response, so the units or aggregators coordinating the DSRs should have instrumentation capable of quickly detecting these excursions and actuating the required response.

The use of frequency responsive load was demonstrated in the Olympic Peninsula project [15]. The project showed that very fast load response to underfrequency events was consistent and effective both at the unit level and in the aggregate. However, the demonstration did not address the question of how many or which loads should be sensitive to frequency. Using the proposed mechanism would permit a TSO to use a market to identify, select and dispatch the required number of frequency-sensitive loads to satisfy the system needs at the lowest cost, without risking a potential over-response from too many loads curtailing during an under-frequency event and settling to a frequency too high to activate the needed secondary reserve response.

\subsection{Performance-based remuneration}

Performance-based remuneration has already been introduced in United States through the FERC Order 755. Similarly, in this work we propose that service providers are paid according to how close they follow the capability parameters they bid to the market when cleared. The estimation of the service provision performance can be done in different ways, depending on which parameters the system operator deems to be the most critical. A service performance index is proposed in [16], where service performance is defined as the root mean square 
error of the actual service delivery compared to an ideal service model:

$$
\begin{aligned}
& \eta=\sqrt{\frac{\sum_{t=0}^{N}\left(Q o S_{t}^{2}\right)}{N}}, \\
& \eta \in[0,1],
\end{aligned}
$$

where $N$ is the time horizon over which the service is delivered and $Q o S \in[0,1]$ is the Quality of Service of the ancillary service. The $Q o S$ is the error in service delivery scaled to the tolerance limits defined by the system operator. When $\eta=0$ the service has been delivered perfectly, and when $\eta=1$, the service delivery is barely acceptable.

Similarly, the reliability parameter defined in the market mechanism can be derived from an average of historical values of the performance metric:

$$
\gamma=1-\frac{\sum_{h=1}^{H} \eta_{h}}{H},
$$

where $H$ is a historical horizon.

\subsection{Example of requirements restructuring}

In this section we present a concrete example of the proposed restructuring. It must be noted that it is a simple example, and a parameterization with complex polytopes and corresponding market clearing is envisioned as future work. This example is also used for the case study in Section 5.

\subsubsection{Parameterization of service performance}

The capability value of a unit is defined through the following parametrization:

$$
\kappa_{i}=g\left(\tau_{i}^{r}, \tau_{i}^{d}, C_{i}\right) \quad \in[0,1]
$$

In the remainder of the paper we define $\tau_{i}^{r}$ as the ramp time, $\tau_{i}^{d}$ as the total duration the unit can sustain the service provision and $C_{i}$ as the power capacity the unit $i$ bids.

\subsubsection{Market mechanism}

The reserve requirement is defined as $T_{t o t}=$ $f\left(\tau_{t o t}^{r}, \tau_{t o t}^{d}, C_{t o t}\right)$. More specifically, for any set of $\left(\tau_{i}^{r}, \tau_{i}^{d}, C_{i}\right)$ values the response function $V_{i}(t)$ is a piecewise linear function and has the following form:

$$
V_{i}(t)= \begin{cases}\frac{C_{i}}{\tau_{i}^{r}} t & \text { if } 0 \leq t \leq \tau_{i}^{r} \\ C_{i} & \text { if } \tau_{i}^{r} \leq t \leq \tau_{i}^{d} \\ 0 & \text { elsewhere }\end{cases}
$$

where $t$ is duration.

Using the above equation, a convex 2-D polyhedra $\mathbb{P}_{i}$ that is bounded by $V(i)$, unit duration $\tau_{i}^{d}$, and $V_{i}(t) \geq 0$ could be constructed as follows:

$$
\mathbb{P}_{i}=\left\{x \in \mathbb{R}^{2} \mid A_{i} x \leq b_{i}\right\}
$$

where $x$ is defined as the vector $[t, V]$ and, $A_{i}$ and $b_{i}$ has the following forms:

$$
A_{i}=\left[\begin{array}{cc}
0 & 1 \\
-\frac{C_{i}}{\tau_{i}^{r}} & 1 \\
1 & 0 \\
0 & -1
\end{array}\right] \quad b_{i}=\left[\begin{array}{c}
C_{i} \\
0 \\
\tau_{i}^{d} \\
0
\end{array}\right]
$$

Market participants respond to the tender request with a bid that consists of: capacity $C_{i}[\mathrm{~kW}]$, bid price $P_{i}^{\text {bid }}$ [\$/MW], ramp time $\tau_{i}^{r}$ [s], duration $\tau_{i}^{d}$ [s] and capability value $\kappa_{i}$; where $i$ denotes the individual bid of each market participant.

The TSO constructs the individual volume $V_{i}$ bid by each market participant and the $V_{i}$ s get adjusted by a reliability parameter $\gamma_{i} . \gamma_{i}$ depends on historical performance of the participating unit. If a unit's error in availability $\left(\epsilon_{i}\right)$ is less than a limit, the unit is eligible for participation. The exemplary market is designed as a single clearing price auction, in which each resources' volume get adjusted by a reliability parameter $\gamma_{i}$, and the clearing price $P_{i}^{\text {clear }}$ gets adjusted by the capability value $\kappa_{i}$. This ensures that the expectation from individual resources is adjusted by its historical average performance, and the total market clearing cost includes the capability value of each resource.

The clearing mechanism identifies a common clearing price based on the most expensive accepted bid, similar to a merit order clearing. Based on the clearing price, the mechanism selects the subset of bids which offer the cheapest overall clearing cost and meet the tender requirements:

$$
\begin{aligned}
& \Omega^{\mathrm{acc}}=\underset{\Omega \in \Omega^{\text {rec }}}{\arg \min } \sum_{i \in \Omega} \kappa_{i} P^{\mathrm{clear}} \\
& \text { s.t. } V_{\text {adjusted }}=\sum_{i \in \Omega^{a c c}} \gamma_{i} V_{i} \\
& \mathbb{P}_{\text {tot }} \subseteq \mathbb{P}_{\text {adjusted }} \\
& P^{\mathrm{clear}}=\max P_{i}^{\mathrm{bid}}, \forall i \in \Omega^{a c c} \\
& \epsilon_{i} \leq \epsilon_{\max }
\end{aligned}
$$

where $\Omega^{a c c}$ and $\Omega^{r e c}$ is the set of accepted and received bids, respectively. $\mathbb{P}_{t o t}$ refers to the polyhedra that is created by the TSO's tender request, and $\mathbb{P}_{\text {adjusted }}$ refers to the polyhedra created by the sum of all units $i$ that are in $\Omega$. 


\subsubsection{Remuneration}

As previously mentioned, remuneration could take performance and capability values into account. An example of a final settlement price can be defined as:

$$
P_{i}^{\text {rem }}=\left(1-\eta_{i}\right) \kappa_{i} P^{\text {clear }} C_{i}, \quad \forall i \in \Omega^{\text {acc }},
$$

which means that each accepted bid gets remunerated the clearing price times the delivered capacity, scaled by the capability value and how close they perform to the promised capability.

\section{Case Study: Primary Frequency Control}

In the case study we show the proposed restructuring of the requirements through an example of primary frequency control. The case uses the example parameterization and market definition presented in Section 4.

Also, in this case the capability value $\kappa$ the TSO assigns to the units is:

$$
\begin{gathered}
\kappa=\alpha_{1} \frac{\tau_{r, 0}}{\max \left(\tau_{r, 0}, \tau_{r, a}\right)}+\alpha_{2} \frac{\min \left(\tau_{d, 0}, \tau_{d, a}\right)}{\tau_{d, 0}} \\
\sum_{i} \alpha_{i}=1
\end{gathered}
$$

where $\tau_{r, 0}$ is the ideal ramp time the TSO desires of the unit and $\tau_{r, a}$ is what the unit can actually deliver. Similarly, $\tau_{d, 0}$ is the ideal delivery duration the TSO desires and $\tau_{d, a}$ is what the unit can deliver. The minimum and maximum functions ensure that units are not overvalued in case they parameters are better than the ideal. In this study case, $\tau_{d}$ and $\tau_{r}$ are valued equally, which means that $\alpha_{1}=\alpha_{2}=0.5$.

\subsection{System setup}

The viability of the proposed market clearing mechanism has been tested through a proof-of-concept study based on an experimental implementation.

The experiment assumes the existence of a market for primary frequency response in which the TSO acts indirectly as a single buyer of services. Based on a calculation of the system need for primary frequency response, the TSO provides a minimum capacity envelope to the market for which the clearing algorithm computes the cost-optimal stacking of resources as described above. The market result is a list of resources to activate, where the most expensive unit sets the clearing price. Each activated resource then continuously provides the service by counteracting frequency excursions until the end of the contracted operating period.

A number of simplifying assumptions have been made for the experiment, without loss of generality. All resources, load as well as generation, are assumed to interact with the market through a single aggregator. The grid is assumed to consist of a single control area overseen by one TSO. No strategic bidding is implemented by the individual resources; base prices remain static. Nevertheless, market results change between operational periods due to the influence of performance evaluations for the individual units.

The operational timeline can be seen in Figure 5 . Shortly before the end of each operational period, the market clears for the upcoming period, and the result is distributed to the aggregator which in turn activates the resources with winning bids. During the operational period, each activated resource provides the service autonomously, based on a local frequency measurement. The power consumption of each resource is continuously measured and the data forwarded to a performance evaluation module. Shortly before the end of each operational period, the performance evaluation result is provided to the market algorithm which clears for the next period, completing the cycle.

The experiment was conducted at the SYSLAB laboratory at DTU Ris $\emptyset$ Campus [17] which features a large variety of DER units coupled to a 16-busbar $400 \mathrm{~V}$ grid. The laboratory has a high degree of automation and facilitates the deployment of distributed control software for cyber-physical experiments in the smart grid domain.

For the experiment, the power system was configured as an island grid, with a $125 \mathrm{kVA}$ Static Frequency Converter (SFC) serving as the grid forming unit. The droop setting of the converter was adjusted to simulate a predetermined frequency response to changes in generation or load.

In order to simplify the experiment, only two physical DER resources were connected to the grid: An $80 \mathrm{~kW}$ load bank to represent all consumption and a $15 \mathrm{~kW}$ redox flow battery to represent all generation. Because the behaviour of the market cannot be studied with such a low number of participants, virtual resources were implemented as real-time simulation processes, each of which would provide an independent frequency response service, responding to measurements of the physical grid frequency conducted every $100 \mathrm{~ms}$. The load bank and the battery were then used to imprint the aggregate power flow of all virtual units onto the physical grid.

Figure 6 shows the entire setup and information flow of the experiment, divided into five layers. The two bottom layers represent the physical setup of the laboratory grid and the DER units used by the experiment. At the third layer, DER component simulators operate based on market input and real-time grid data. The simulation output is then mapped to the physical DER units (i.e. the load bank and the battery). The layer above performs 


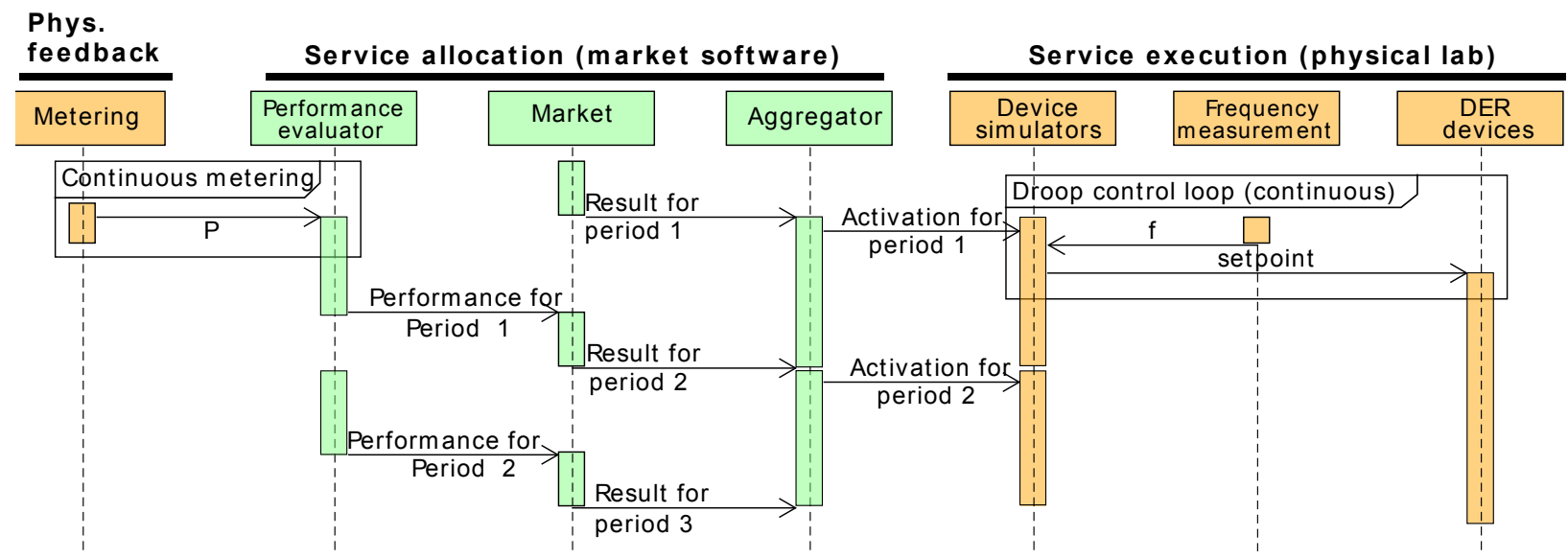

Figure 5: Interactions between system components (time advances down the vertical axis).

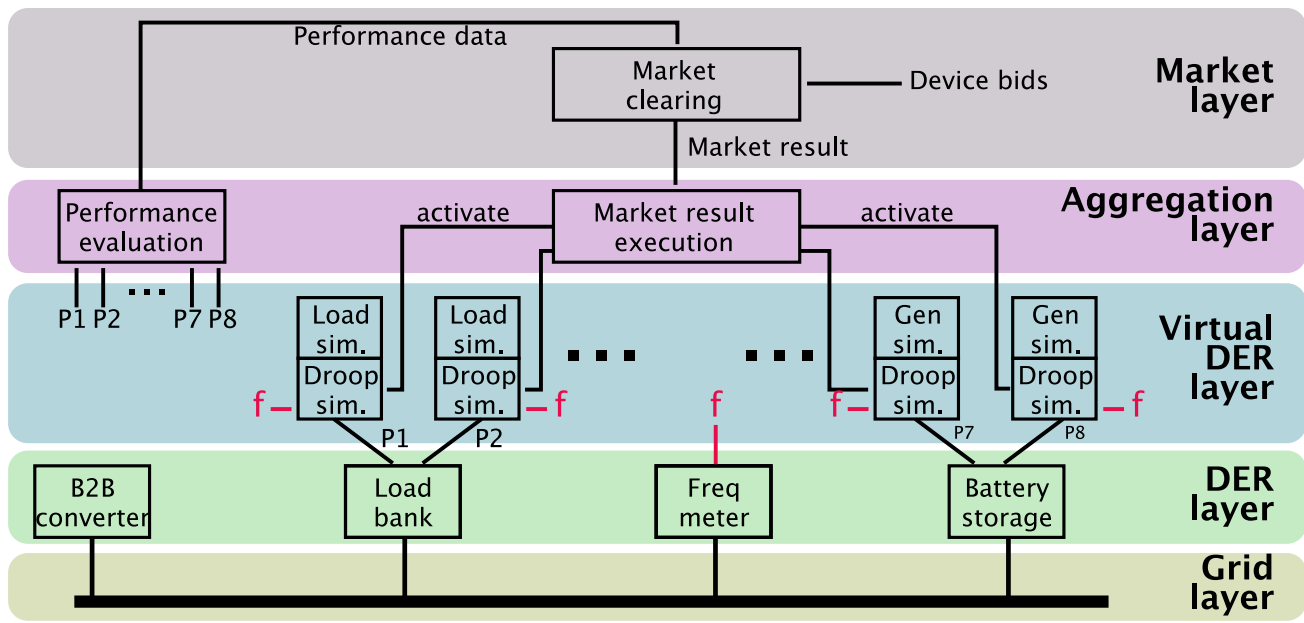

Figure 6: Overview of the experiment setup.

aggregation and evaluation of performance data and distribution of market results, interacting directly with the market layer at the top.

\subsection{Experiment description}

A set of eight fictitious market participants-three generators and five loads-was generated as given in Table 1. At the start of the experiment, a net load of $17 \mathrm{~kW}$ had been established as the steady-state baseline, resulting from the superposition of $27 \mathrm{~kW}$ of consumption and $10 \mathrm{~kW}$ of generation. The droop characteristics of the back-to-back converter had been adjusted to provide an output frequency of $50 \mathrm{~Hz}$ at this load point. Additional generation and consumption capacity is used to obtain a symmetrical reserve assignment of $\pm 27 \mathrm{~kW}$ such that $44 \mathrm{~kW}$ of consumption causes the converter output to drop to $48 \mathrm{~Hz}$, while $10 \mathrm{~kW}$ of generation raises it to $52 \mathrm{~Hz}$. Additional loads in the physical grid are used to introduce step disturbances during the experiment.

The system need $V_{\text {tot }}$ was characterized by $\tau_{\text {tot }}^{r}=6$ seconds, $\tau_{t o t}^{d}=60$ seconds, and $C_{t o t}=12 \mathrm{~kW}$. During the experiment, the market cleared every 60 seconds with the same eight participants. The clearing is done through an exhaustive search that tests all possible service provider combinations to form the cheapest reserve.

In the period between clearings, step disturbances were applied to the physical grid, in order to determine the reaction of individual market participants. $\eta$ and $\gamma$ were continuously calculated for each participant in order to see the impact of performance based adjustments in (12) on market clearing results.

\subsection{Results}

In Figure 7, we present the results of an example run of the new market clearing mechanism and compare it with a traditional merit order approach as used in the Nordic 
Table 1: Market players and their respective assets. The quoted price is only indicative of the relative cost between the units.

\begin{tabular}{lllllll}
\hline Unit & Owner & Resource type & Baseline & Capacity & Ramp time & Duration \\
\hline$P_{1}$ & Bidder 1 & load & $5 \mathrm{~kW}$ & $5 \mathrm{~kW}$ & $3 \mathrm{~s}$ & $120 \mathrm{~s}$ \\
$P_{2}$ & Bidder 1 & load & $6 \mathrm{~kW}$ & $4 \mathrm{~kW}$ & $4 \mathrm{~s}$ & $80 \mathrm{~s}$ \\
$P_{3}$ & Bidder 2 & generation & $3 \mathrm{~kW}$ & $2 \mathrm{~kW}$ & $2 \mathrm{~s}$ & $9 \mathrm{X} / \mathrm{kW}$ \\
$P_{4}$ & Bidder 2 & generation & $3 \mathrm{~kW}$ & $2 \mathrm{~kW}$ & $3 \mathrm{~s}$ & $10 \mathrm{X} / \mathrm{kW}$ \\
$P_{5}$ & Bidder 2 & load & $5 \mathrm{~kW}$ & $4 \mathrm{~kW}$ & $4 \mathrm{~s}$ & $60 \mathrm{~s}$ \\
$P_{6}$ & Bidder 2 & load & $6 \mathrm{~kW}$ & $4 \mathrm{~kW}$ & $1 \mathrm{~s}$ & $20 \mathrm{~s}$ \\
$P_{7}$ & Bidder 2 & generation & $4 \mathrm{~kW}$ & $1 \mathrm{~kW}$ & $3 \mathrm{~s}$ & $110 \mathrm{~s}$ \\
$P_{8}$ & Bidder 2 & load & $5 \mathrm{~kW}$ & $5 \mathrm{~kW}$ & $1 \mathrm{~s}$ & $130 \mathrm{~s}$ \\
\hline
\end{tabular}

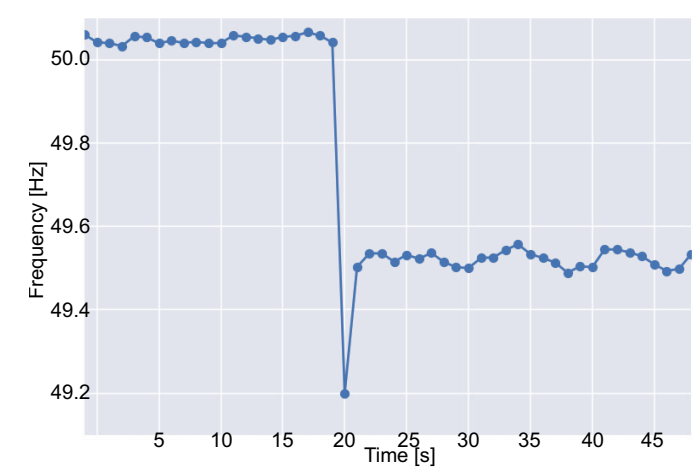

Figure 8: Response to a $10 \mathrm{~kW}$ disturbance.

System for the Manual Regulating Power Market. This approach consists of creating a list of all received bids (which pass the minimum requirements) sorted by the cheapest price first, and then accepting bids sequentially until the required capacity is acquired.

It is clear that both mechanisms select sets of participants that can satisfy the system need. Since some units that are cleared by the new methodology (e.g., Bidder 2/Bid:5/ $\mathrm{P}_{7}$ ) do not satisfy the requirements imposed by traditional merit order approach, it is possible to see that the merit order results in a set with higher capacity and longer duration than needed. In this case, the total capacity cost for the new market clearing mechanism is $30 \%$ less than the merit-order case. Note that, the merit-order solution is in the solution space of the new market clearing methodology. However, due to relaxed requirements, a less costly option is available in this case.

To demonstrate the response and frequency arrest capability of the selected resources we introduced a $10 \mathrm{~kW}$ load increase as shown in Figure 8. As it can be seen in the frequency behavior in Figure 8, the units that have been selected have successfully responded to the disturbance and arrested the frequency at $49.5 \mathrm{~Hz}$. Note that, the back-to-back converter does not have an inertia, and the response from units results in a new frequency equilibrium. It must also be noted that clearing the market through an exhaustive search does not scale, and the market formulation might need adjustment in order to be cleared by scalable algorithms.

\section{Conclusion}

This work introduces the key concept of parameterizing ancillary services (AS) requirements, so that these become a multi-dimensional product. By doing this, TSO/RTOs can assign a value to different technologies depending on how they contribute to needed system reserves, e.g., a very slow ramping unit that can sustain a response over long duration can complement a fast ramping unit with short duration. Since both units contribute equally to the reserve, yet along different product parameters, they may have similar capability value towards the TSO.

The case study carried out in the laboratory showed that the proposed restructuring can lower the cost of acquiring and activating reserves. As the amount of intermittent renewable energy production increases in the power system, TSOs must find new ways of keeping a balanced and reliable grid. The capability value and the service parameterization presented in this work can be used by the TSOs to form reserves that utilize the benefits and synergies of different technologies, and thereby better address the new operational requirements of the power system.

Future work includes exploring alternative market formulations, larger simulation studies, e.g. using the WECC 2024 base case, the optimization of the clearing algorithm to improve scalability and a more thorough comparison of the proposed solution with existing market mechanisms and product/service definitions.

\section{Acknowledgments}

Parts of this work are supported by the Programme for Energy Technology Development and Demonstration (EUDP) through PowerLabDK, Innovation Fund Denmark through the iPower project and the European Union 

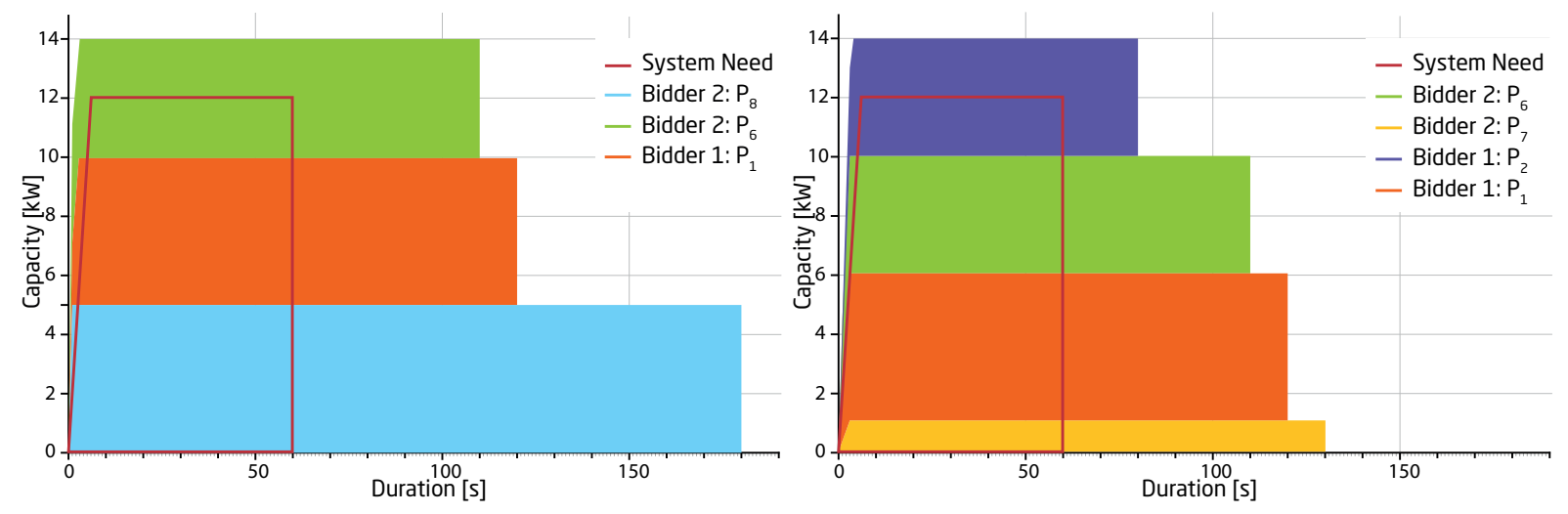

Figure 7: Comparison of merit order approach (left) with new market clearing mechanism $\forall \gamma=1$ (right).

through the Researcher Exchange Programme of the FP7 ELECTRA project.

SLAC National Accelerator Laboratory and the Lawrence Berkeley National Laboratory are operated for the US Department of Energy by Stanford University under Contract No. DE-AC02-06-76SF00515 and by the University of California, Berkeley under Contract No. DE-AC02-05CH11231, respectively.

\section{References}

[1] EURELECTRIC, "A sector in transformation: electricity industry trends and figures," tech. rep., Jan 2015.

[2] B. Kirby, "Load response fundamentally matches power system reliability requirements," in Power Engineering Society General Meeting, 2007. IEEE, pp. 1-6, June 2007.

[3] P. Cappers, J. MacDonald, C. Goldman, and O. Ma, "An assessment of market and policy barriers for demand response providing ancillary services in us electricity markets," Energy Policy, vol. 62, pp. 1031-1039, 2013.

[4] M. Norton, H. Vanderbroucke, E. Larsen, C. Dyke, S. Benares, C. Latour, and T. V. Van, "Demand Side Response Policy Paper," tech. rep., European network of transmission system operators for electricity (ENTSO-E), 2014.

[5] Y. Rebours, A Comprehensive Assessment of Markets for Frequency and Voltage Control Ancillary Services. PhD thesis, School of Electrical and Electronic Engineering University of Manchester, 2008.

[6] European network of transmission system operators for electricity (ENTSO-E), "Network code on loadfrequency control and reserves," tech. rep., 2013.

[7] NERC Resources Subcommittee, "Balancing and frequency control - a technical document," tech. rep., North American Reliability Corporation, 2011.

[8] ENTSO-E, "Continental europe operation handbook - p1 load-frequency and control performance," tech. rep., European network of transmission system operators for electricity, 2014.
[9] J. H. Eto, J. Undrill, P. Mackin, R. Daschmans, B. Williams, B. Haney, R. Hunt, J. Ellis, H. Illian, C. Martinez, M. O'Malley, K. Coughlin, and K. H. LaCommare, "Use of frequency response metrics to assess the planning and operating requirements for reliable integration of variable renewable generation," Tech. Rep. LBNL-4142E, Lawrence Berkeley National Laboratory, 2010.

[10] Energinet.dk, "Ancillary services to be delivered in Denmark - Tender Conditions," tech. rep., 2012.

[11] Smart Energy Demand Coallition (SEDC), "Explicit demand response in europe - mapping the markets 2017," tech. rep., 2017.

[12] E. Vrettos, C. Ziras, and G. Andersson, "Integrating large shares of heterogeneous thermal loads in power system frequency control," in PowerTech Conference, Eindhoven, Netherlands, pp. 1-6, ETH Zurich, 2015.

[13] Y. V. Makarov, S. Lu, J. Ma, T. B. Nguyen, C. E. Commission, et al., Assessing the value of regulation resources based on their time response characteristics. Pacific Northwest National Laboratory, 2008.

[14] "PJM manual 11: energy \& ancillary services market operations." Available online at www.pjm.com/\$ sim\$/media/documents/manuals/m11.ashx. last accessed 15.06.2017.

[15] D. J. Hammerstrom, J. Brous, D. P. Chassin, G. R. Horst, R. Kajfasz, P. Michie, T. V. Oliver, T. A. Carlon, C. Eustis, O. M. Jarvegren, et al., "Pacific northwest gridwise testbed demonstration projects; part ii. grid friendly appliance project," Tech. Rep. PNNL-17167, Pacific Northwest National Laboratory, Richland, Washington (USA), 2007.

[16] D. E. M. Bondy, A. Thavlov, J. B. M. Tougaard, and K. Heussen, "Performance requirements modeling and assessment for active power ancillary services," 2017. To be published in the proceedings of PowerTech 2017.

[17] DTU, "SYSLAB: Technical Specifications." Available online at http://www.powerlab. $\mathrm{dk} /$ facilities/syslab/technicalspecifications. 